\title{
Pappus in optical space
}

\author{
JAN J. KOENDERINK, ANDREA J. vAN DOORN, and ASTRID M. L. KAPPERS \\ University of Utrecht, Utrecht, The Netherlands \\ and \\ JAMES T. TODD \\ Ohio State University, Columbus, Ohio
}

\begin{abstract}
Optical space differs from physical space. The structure of optical space has generally been assumed to be metrical. In contradistinction, we do not assume any metric, but only incidence relations (i.e., we assume that optical points and lines exist and that two points define a unique line, and two lines a unique point). (The incidence relations have generally been assumed implicitly by earlier authors.) The condition that makes such an incidence structure into a projective space is the Pappus condition. The Pappus condition describes a projective relation between three collinear triples of points, whose validity canin principle-be verified empirically. The Pappus condition is a necessary condition for optical space to be a homogeneous space (Lobatchevski hyperbolic or Riemann elliptic space) as assumed by, for example, the well-known Luneburg theory. We test the Pappus condition in a full-cue situation (open field, broad daylight, distances of up to $20 \mathrm{~m}$, visual fields of up to $160^{\circ}$ diameter). We found that although optical space is definitely not veridical, even under full-cue conditions, violations of the Pappus condition are the exception. Apparently optical space is not totally different from a homogeneous space, although it is in no way close to Euclidean.
\end{abstract}

Physical space is defined via measurements using yardsticks, measuring tapes, theodolites (a surveying instrument), and so forth, whereas optical space is defined via judgments of human observers depending solely on eye measure. It is well known that optical space differs from physical space. Three-dimensionalEuclidean space is a very good model for physical space (Coxeter, 1961), whereas the structure of human optical space is still a matter of debate. It is well known that judgments of Euclidian properties are often far from veridical but typically show systematic deviations. Since the beginning of serious psychophysics (second half of the 19th century), various aspects of the structure of human optical space have been discussed (Hauck, 1879; Helmholtz, 1866). Helmholtz was the first to seriously consider a Riemannian structure. Since the mid-20th century, it has been frequently suggested that human optical space is a Riemannian space and very likely one of the classical homogeneous spaces - that is to say, elliptical space (Riemann's elliptical plane), Euclidean space, or hyperbolical or Lobatchevskian space. The major reason for this view has been the seminal work of Luneburg (1947), inspired by classical data gathered by many authors (Blumenfeld, 1913; Helmholtz, 1866; Hillebrand, 1929). Apart from the empirical evidence, Luneburg was mainly led by theoretical considerations (see Gibson,

Correspondence concerning this article should be addressed to J. J. Koenderink, Helmholtz Instituut, Universiteit Utrecht, Buys Ballot Laboratory, Princetonplein 5, P. O. Box 80.000, 3508TA Utrecht, The Netherlands (e-mail: j.j.koenderink@ phys.uu.nl).
1950). He considered the Riemannian structure to be selfevident. Likewise, he accepted congruence as intuitively certain, in no need of empirical verification. This is a crucial assumption, since congruence (that things may "look metrically identical" despite arbitrarily large variations in location and attitude) implies homogeneity. Once these properties are granted, human optical space has to be one variety of the classical spaces: elliptical, Euclidean, or hyperbolical. The only uncertainty left then is the magnitude of the curvature. This indeed was Blank's (1958a) notion of psychophysics of human optical space to determine the curvature of human optical space.

It seems to us that the matter of the structure of human optical space remains effectively open. It should even be a point of debate whether an optical space that has a fixed relation to physical space independent of what is in it has any basis in fact at all. The problem seems (to us) to be primarily empirical. Various aspects allow empirical exploration. It seems to us that no such possibilities should be neglected. In designing programs of research, one may take advantage of the various "ways to Euclidean geometry" as documented in mathematics. Perhaps the more important of these is Klein's $(1932,1939)$ Erlanger Programm. Klein understood Euclidean structure to be the pinnacle of a hierarchy of progressively "less primitive" geometries, the weakest geometry being topological space. (If mere sets-bags of marbles, say-have a claim to the title of "geometry" at all, topological spaces are the most primitive). Topological properties are conserved under arbitrary deformations that conserve local neighborhoods; hence the popular term rubber sheet geometry (Coxeter, 1961). 
Each progression toward Euclidean geometry is defined through the narrowing down of the transformation group that leaves the geometrical entities invariant. Projective geometry conserves points, lines, and their incidence properties. The transformation group is that of collineations. Affine geometry does the same, but in addition conserves parallellism and bisection of line segments. The transformation group is that of affinities (general linear transformations). Similarity geometry does all that and in addition conserves angles. The transformation group is the similarities. Finally, Euclidean geometry does all this and in addition conserves lengths. The transformation group is the isometries (Euclidean congruences). Notice that isometries are similarities, which are affinities, which are collineations, which are isomorphisms (topological transformations). Thus Euclidean geometry is a specialization of affine geometry, which is, again, a specialization of projective geometry. If optical space fails to be Euclidean, might it be affine, or, failing that, projective?

Riemannian spaces stand somewhat outside of the Kleinian scheme. They are defined via their local metrical properties; global properties are then defined via integration over local domains. The straight lines of Riemann spaces are geodesics, curves of extremal length between any two points. In general, (nonhomogeneous spaces), these geodesics fail to form a nice projective nexus, as was proven by Beltrami (1865). Luneburg and Blank took homogeneity very much for granted, though the modern empirical evidence (Indow, 1990, 1991, 1997) clearly supports nonhomogeneity. This is an empirically decidable issue.

In this paper, we investigate the problem of whether human optical space possesses a projective structure, taking its incidence structure (see below) for granted. Of course, even the incidence structure is up for grabs, but for the purpose of this paper, we simply assume it. In doing this, we assume less than is usual. For instance, Luneburg (1947) does not even so much as mention that he assumes projective structure (though in fact he does).

An incidence structure is built upon sets of points and lines. One assumes that any two distinct points determine a unique line and that any two distinct lines determine a unique point. When the sets of points and lines are sufficiently large (axioms of continuityand order), one obtains a partial characterization of Euclidean space (clearly Euclidean space is an incidence geometry with much additional structure). In order to approximate Euclidean space even closer, one needs to exclude certain "pathologies" via additional assumptions. In this paper, we assume the incidence structure of human optical space and investigate whether certain pathologies can be empirically excluded (at least approximately).

Important assumptions that turn incidence geometry into projective geometry (Blumenthal, 1995) are the Desargues and Pappus properties. ${ }^{1}$ Of these, the Pappus property is the strongest in the sense that the Desargues property follows from the Pappus property and the incidence structure. Curiously, it makes a difference whether one studies projective (3-D) space or the projective plane: In three-dimensional space, one may prove (Bennett, 1995; Blumenthal, 1995) Desargues from the axioms of incidence (thus, it is a theorem and need not be added as an axiom). In the case of the projective plane, one can prove Desargues when Pappus is taken as an axiom. The Pappus property ensures commutativity of multiplication (Bennett, 1995; Blumenthal, 1995). (Planes where the Pappus property fails to hold are noncommutative geometries that are of interest for their own sake. When Pappus is not empirically verified for human optical space, such general geometries immediately become candidate models for human optical space.)

\section{The Pappus Property}

Pappus was an Alexandrian geometer (born 350 AD) who proved the following remarkable Euclidean theorem (see Figure 1):

Assume $\left\{P_{1}, P_{2}, P_{3}\right\}$ and $\left\{Q_{1}, Q_{2}, Q_{3}\right\}$ are collinear triples of points. Define $\left\{R_{1}, R_{2}, R_{3}\right\}$ as the intersections of the lines defined by the point pairs $\left\{\left\{P_{1}, Q_{2}\right\},\left\{P_{2}, Q_{1}\right\}\right\}$, $\left\{\left\{P_{1}, Q_{3}\right\},\left\{P_{3}, Q_{1}\right\}\right\}$ and $\left\{\left\{P_{2}, Q_{3}\right\},\left\{P_{3}, Q_{2}\right\}\right\}$, respectively. Then the triple $\left\{R_{1}, R_{2}, R_{3}\right\}$ is collinear.

In appreciating this theorem, one has to remember that it is very remarkable for any three points to be collinear. Any triple of points selected at random has a probability of 1 to be noncollinear(or in general position). Pappus thus yields another such remarkable triple given any pair of them. What is important here is that Pappus's theorem cannot be proved from the incidence structure. When one adds Pappus (as an axiom) to the incidence structure, one obtains what is known as projective geometry. Projective geometry is the royal road to the classical non-Euclidean geometries and eventually leads to Euclidean geometry itself. Thus an empirical verification of the Pappus property goes a long way to establish Luneburg's (1947) ad hoc assumption as an empirical fact. This has not been attempted before, nor has the Desargues property been empirically tested. (Although Suppes, 1977, mentions the experiment by Foley, 1964, this is really a test of the Pasch axiom, which is a clever way to enforce the existence of planes in projective space; see Bennett, 1995.)

Pappus's theorem is known to hold for the classical homogeneous spaces (see Figure 2), which are the spaces of constant Gaussian curvature (Beltrami, 1865, 1868) (in the remainder of this paper, space will typically denote twodimensional space)—namely, (1) the hyperbolic plane of Lobachevski and Bolyai, which has constant negative curvature; (2) the Euclidian plane of old, which has vanishing curvature; and (3) Riemann's elliptical plane, which has constant positive curvature.

The hyperbolic plane was considered the most likely model for human optical space by Luneburg, although his congruency argument only serves to single out the homogeneous space from all Riemann spaces. An empirical verification of the Pappus property amounts to a check of homogeneity once the Riemannian structure is granted (Beltrami, 1868). 


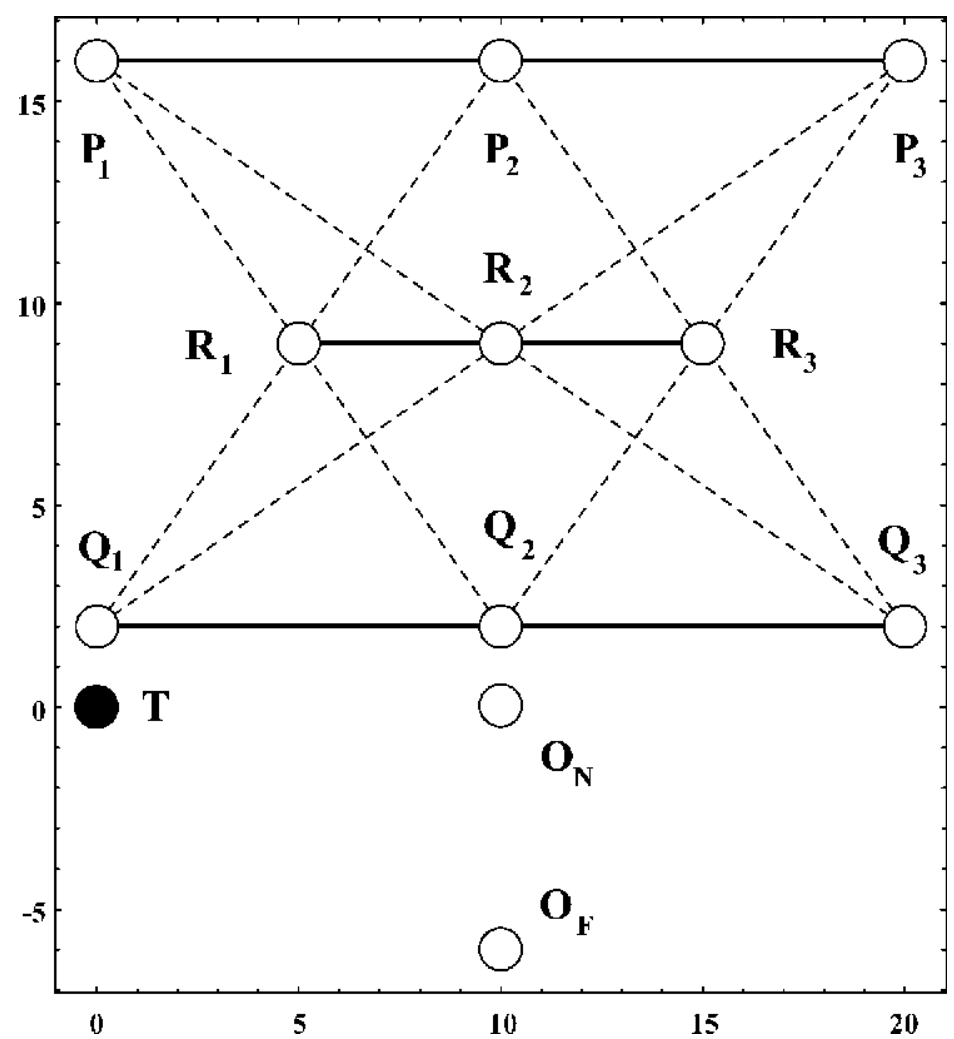

Figure 1. The geometrical layout of the configurations used in this study. The observer is either at $O_{\mathrm{N}}$ ("near") or at $O_{\mathrm{F}}$ ("far"). The points $P_{1}, P_{3}, Q_{1}$, and $Q_{3}$ are fixed. The points $P_{2}$ and $Q_{2}$ are tentative: Both are in the forward direction and collinear (in visual space!) with, respectively, $P_{1}, P_{3}$ and $Q_{1}, Q_{3}$. Their positions were determined in the initial phase of the experiment. The points $R_{1}$, $R_{2}$, and $R_{3}$ are defined by the Pappus construction in visual space and were determined in the second phase of the experiment. The geodesic measurement station was located at $T$. All measures are in meters.

\section{Empirical Investigation of the Pappus Property}

In this paper, we investigate the empirical validity of the Pappus property. The conditions under which the experiments were conducted differ greatly from established practice. Classically, one has been mainly interested in binocular vision. This is because of the conviction that monocular optical space is confined to the visual field, since depth perception is the domain of binocularity. This is mainly an a priori notion that disregards the empirical fact that monocular observers or animals that (almost) lack binocular overlap are not particularly handicapped in their spatial behavior. In order to enforce binocularity, one brings observers into a dark room and confronts them with a few (as few as will enable the task to be done) dim luminous points. Usually, the observer's head is fixed and the field of view is severely restricted. Although such methods have revealed much that is of interest from an academic perspective, it may be doubted whether such an approach contributes to the understanding of optical space in daily life.

In the classical setup, the geometry has typically been limited to a plane through the eyes. One dimension of such a plane is also a dimension of the visual field, the other is depth. In our case, the full-cue situation, the observer is aware of a structured, three-dimensional visual world. We studied the validity of the Pappus property in the horizontal plane at eye height (the "horizon" of the human observer), but unlike in the classical case, this plane obtains its structure from the very fact that it is an integral part of the observer's visual world. In fact, although we will continue to use the term horizontal plane at eye height, this is merely for convenience, because an infinite number of other (nonvertical) planes possess the same structure. It is perhaps more apt to conceive of this entity as an abstract two-dimensional object instead of a concrete plane. In more formal terms, we would rather speak of the visual world (three-dimensional) modulo the vertical dimension (and thus a two-dimensional object, such as a plane). The depth dimension of this plane derives its structure from the visual world, among more due to monocular cues like position on the ground plane, and so forth.

In our experiments, the observers were confined to a fixed station point, in the sense that they had to keep their 

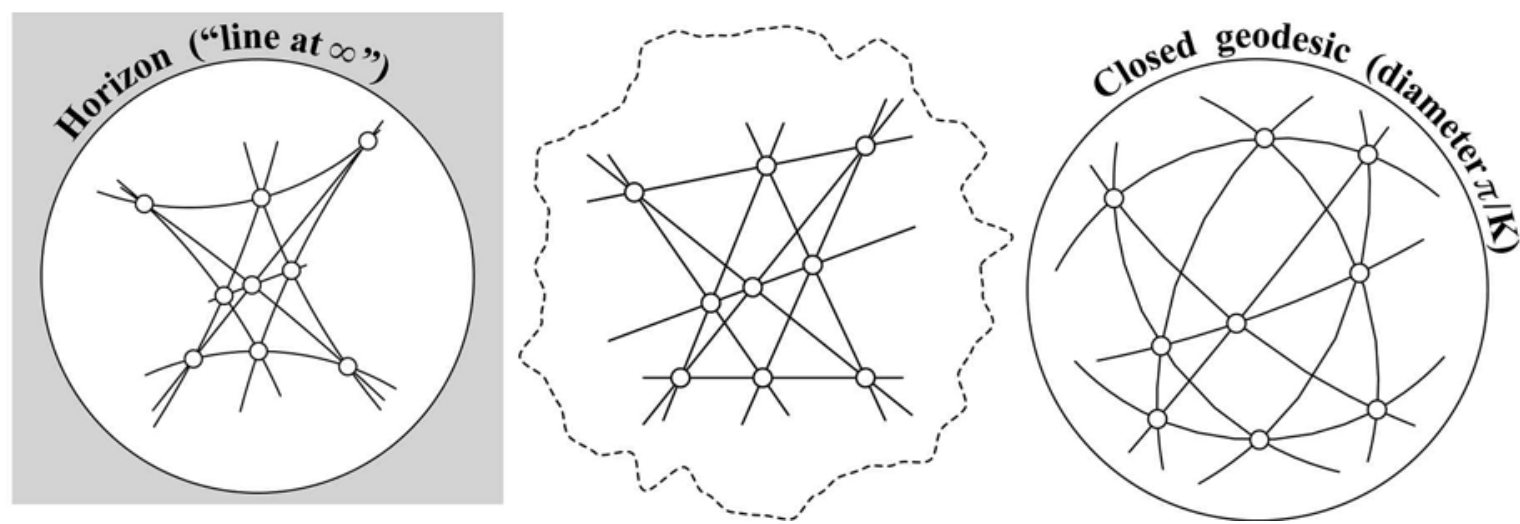

Figure 2. Pappus configuration in the three classical homogeneous spaces (Hilbert \& Cohn-Vossen, 1932). Left: In the Kleinean model of the hyperbolic plane (Lobachevski's absolute geometry), the angle sums of all triangles are less than $\mathbf{1 8 0}^{\circ}$, the curvature is negative. This space is infinite, though the model represents the line at infinity as a circle. Center: The Euclidean plane is infinitely extended, the angle sum in all triangles equals $\mathbf{1 8 0}^{\circ}$, thus the curvature vanishes everywhere. Right: Here a northern hemisphere is stereographically mapped onto a circular disk. Since angles are conserved, one can see that the sum of interior angles exceeds $180^{\circ}$ for all triangles. The example of the equator shows that the geodesics (straight lines) are closed in this space. Their diameter depends on the curvature $K$. In all three spaces, the curvature is constant (hence, homogeneous spaces), and the Pappus theorem holds. The projective structure (incidence relations and Pappus) is the same in all three cases.

feet within a 30-cm diameter region and remain upright at all times (fixed eye height). They were quite free to make head and eye movements, to twist at the waist, or even to change their stance. The environment was a large lawn under bright daylight conditions. The freedom granted to the observer made it practical to use fields of view that enormously exceeded the classical ones (up to $160^{\circ}$ ). In many cases, the observers were unable to see the configurations at a single glance and had to make bodily movements in order to be able to perform the task at all. This is important because we expect possible violations of the Pappus condition especially for very large configurations. (Continuity enforces the projective structure in the small.) Perhaps regrettably, these conditions preclude a meaningful confrontation with the classical data (e.g., "It cannot be emphasized too strongly that experiments conducted without these precautions, i.e., tiny points in the dark, fixed head, etc., cannot be expected to give results comparable to those cited here"; Blank, 1953).

In order to perform experiments in optical space one needs certain assumptions that link human optical space to physical space. After all, the configurations, even those in optical space, can only be defined via physical structures. We assume that (1) points in optical space correspond in a one-to-one fashion to points in physical space, at least to the resolution demanded by the experiment; and (2) egocentric directions (straight lines through the origin in optical space) correspond in a one-to-one fashion to lines through the vantage point (midpoint of the interocular segment, say) in physical space. Both assumptions are shots in the dark. However, similar assumptions have been (implicitly) made by all previous authors. Since we assume an incidence structure for optical space, a line in optical space is (by assumption) defined by any two of its points (distinct of course). Since points in optical and physical space correspond, this means that we may define a line in optical space through two points (physical landmarks!) in physical space.

In order to investigate the Pappus property we have to be able to (1) prepare collineartriples of points in optical space, (2) construct the intersection of two lines in optical space, and (3) check collinearity for any triple of points in optical space. Here is how this can be operationalized:

In order to prepare a collinear triple of points in optical space $\left\{P_{1}, P_{2}, P_{3}\right\}$, say, we start by defining two points $\left\{P_{1}, P_{3}\right\}$, say, via physical landmarks. The problem is to place the point $P_{2}$ on the line $P_{1}-P_{3}$. It is important to grasp the fact that this cannot conceivably be done with conventional geodesic methods, since these can only establish collinearity in physical space. The way to find a point, $P_{2}$, is to place a physical landmark, $\bar{P}_{2}$, in the general direction of $P_{2}$, thus defining the line $\varepsilon-\bar{P}_{2}$ in optical space through the egocenter $\varepsilon$. Then we ask the observer to intersect the lines $P_{1}-P_{3}$ and $\varepsilon-\bar{P}_{2}$ (both in optical space!), thus obtaining a point $P_{2}$ in optical space that is collinear with $P_{1}-P_{3}$. To $P_{2}$ in optical space there corresponds a point (landmark!) in physical space. This solves the problem.

In order to check collinearity of three points in optical space $\left\{R_{1}, R_{2}, R_{3}\right\}$, say, we follow a similar scheme. First, we place a landmark $R_{2}$ on the line $\varepsilon-R_{2}$ (here several switches between points in optical and physical space occur!). Then we ask the observer to find the intersection $R_{2}^{\prime}$ of the lines $\varepsilon-\bar{R}_{2}$ and $R_{1}-R_{3}$ (again, mind the switches!). Next we interpret the points $R_{2}$ and $R_{2}^{\prime}$ as points in physical space and check their physical coincidence. Of course, this involves some statistical procedure (see below). When coincidence obtains in physical space, the triple of points $\left\{R_{1}, R_{2}, R_{3}\right\}$ in optical space is-by construction-collinear. This solves the problem.

Notice that the viability of these operationalizationsdepends critically on the possibility of performing two tasks 
that the observer has to do by eye measure: (1) to find the intersection of an egocentric direction (as defined by a single landmark) with an optical line as defined by a pair of landmarks; and (2) to find the intersection of two optical lines, each defined by a pair of landmarks.

In practice, we asked the observer to place a landmark at the intersection in order to be able to trace the intersection in physical space. Fortunately, we have found (see below) that human observers find this task quite natural. Then, the remaining task (left to the experimenter) is simply to find the locations of the various landmarks by any method of classical geodesy.

Notice that the verification of the Pappus property for a given configuration involves a rather long sequence of such operations (see Figure 1):

Select point pairs $\left\{P_{1}, P_{3}\right\}$ and $\left\{Q_{1}, Q_{3}\right\}$ and egocentric directions $\varepsilon-\bar{P}_{2}$ and $\varepsilon-\bar{Q}_{2}$;

Find collinear triples $\left\{P_{1}, P_{2}, P_{3}\right\}$ and $\left\{Q_{1}, Q_{2}, Q_{3}\right\}$, where $P_{2}$ is the intersection of $P_{1}-P_{3}$ with $\varepsilon-\bar{P}_{2}$ and similar for $Q_{2}$;

Find the intersections of $\left\{\left\{P_{1}-Q_{2}\right\},\left\{P_{2}-Q_{1}\right\}\right\}$ (defines $R_{1}$ ), $\left\{\left\{P_{2}-Q_{3}\right\},\left\{P_{3}-Q_{2}\right\}\right\}$ (defines $R_{3}$ ), and $\left\{\left\{P_{1}-Q_{3}\right\},\left\{P_{3}-Q_{1}\right\}\right\}$ (defines $R_{2}$ );

Check the collinearity of $\left\{R_{1}, R_{2}, R_{3}\right\}$.

This sequence of operations suffices to check the Pappus property for a single pair of collinear triples of points. Of course, one cannot hope to repeat similar checks for any such pair of arbitrary collinear triples, since there exist multiple infinities! In this experiment, we have investigated only two configurations. Though admittedly very limited, it is, to the best of our knowledge, the first such check to be reported in the literature and thus perhaps of some interest.

Notice that the final check is one of coincidence of two landmarks in physical space. Both landmarks are arrived at via some intricate psychophysical procedure. Clearly, the probability of exact coincidence is nil. Indeed, this is trivially the case since even the best state of the art geodesy will involve some (however small) finite tolerance. Thus, the Pappus condition will always be violated in the strict sense. However, when the magnitude of this violation can be explained by the expected scatter in the empirical results, we are justified in saying that "the Pappus condition is empirically verified," because that is simply another way of saying that the violation is not significant. Clearly, the final check has to be a statistical one.

\section{Methodological Problems}

Optical, or visual, space differs from physical space in many respects. Visual space in the normal, outdoor environment is typically strongly dominated by the ground plane and the direction of gravity. Objects are located either with respect to some other, more dominant objects (like leaves on a tree) or with respect to the ground plane. The structure of the ground plane is partly due to the structure of the visual field, partly due to the perception of depth, which is the subjective correlate of physical range. Especially in the far field, the ground plane is apparently highly compressed due to perspective, and monocular depth cues dominate perception. Even though we nominally investigate the geometry of the horizontal plane at eye height, the ground plane is still important in the full-cue situation. This is because all entities - say, the stakes - are placed on the ground plane and are visible over their full extent, even if the instruction is to attend to the horizon plane.

\section{METHOD}

Observers directed a radio-controlled vehicle to various positions in the environment defined by the intersection of implicitly defined lines in optical space. These lines were either defined by pairs of physical landmarks or by the egocentric direction toward a single physical landmark. In no case were there more than the number of landmarks strictly necessary to define the given task visible.

The observers were not allowed to see the crew of experimenters change landmarks in the field. The experimenters used walkie-talkies to communicate, inaudible to the observers. Of course the observers were kept in total ignorance of the actual configurations. Debriefing a posteriori revealed that no observer figured out the full Pappus configuration. They simply tried to set the points of intersection for a number of apparently random lines to the best of their abilities.

\section{Environment}

The experiment was conducted on a large, well-kept lawn, the grass being somewhat less than ankle high. Trees and buildings were visible in various directions, the free space available was about $80 \times$ $80 \mathrm{~m}$. During the experiment, which was conducted during the summer months in the Netherlands (latitude about $54^{\circ}$ ) during the day time (between $0900 \mathrm{~h}$ and $1700 \mathrm{~h}$ ), fair weather conditions persisted. Of course, the sun moved through the sky during the day and cloud coverage (although generally low) was variable.

\section{Observers}

The 4 observers were young adults of both sexes, were naive to the purpose of the experiment, and were paid for their efforts. They had normal or corrected-to-normal visual acuity, normal binocular stereopsis, and no known visual abnormalities. They were given a short training session in order to gain some familiarity in driving the radio-controlled vehicle. The training did not involve the Pappus configuration but was concentrated on negotiating angles, turning circles around poles, and driving slaloms.

\section{Geodesy}

We used a laser range finder combined with a conventional theodolite to measure locations in the field from a single station point. The theodolite-range finder geodesic station was located outside the region involving the Pappus configuration and the observer. The theodolite allowed us to measure angles with $0.001^{\circ}$ accuracy and the laser range finder to measure ranges with $0.001 \mathrm{~m}$ accuracy. Overall, we were able to obtain accuracies in the millimeter range over distances of up to $100 \mathrm{~m}$. We used conventiona $12 \mathrm{~m}$ length red and white stakes as static physical landmarks. A vehicle (about half a meter long and quarter meter high) carried a pole with an $8 \mathrm{~cm}$ edge length cube on top (at $155 \mathrm{~cm}$, i.e., about eye height) and acted as a moveable landmark.

During the experiment, various geodesic measurements and calculations had to be performed because part of the configuration was based on the observer's eye measure.

\section{Task}

The observer had to perform one of two tasks in sequence. One task was to intersect a line segment defined by its two endpoints 
marked by stakes placed in the ground with an egocentric direction defined by a single stake. The other task was to intersect two line segments, each defined by a pair of stakes. The observer performed these tasks by directing the vehicle carrying the pole to the intersection (by eye measure) under radio control. The explicit instructions were to place the cube on top of the pole mounted on the vehicle at the intersection at eye height. Since the lawn was quite uneven on the scale of the vehicle, the pole was rarely exactly vertical, and the instruction to place the cube on top was a crucial one. The geodesic measurements always involved the location of the cube, not the ground position of the vehicle.

In many cases, the task involved landmarks that could not be seen at a single glance, but required body movements in order to be seen. For instance, in one condition, the two landmarks that defined a line were as far as $160^{\circ}$ apart in the visual field. (See Figure 3.)

\section{EXPERIMENTS}

Verification of Pappus requires that the observer carries out instances of each of these tasks thrice. That is to say:

一place $P_{2}$ on $P_{1} P_{3}$,

-place $Q_{2}$ on $Q_{1} Q_{3}$, -intersect $P_{1} Q_{2}$ with $P_{2} Q_{1}$ (yields $R_{1}$ ),

-intersect $P_{2} Q_{3}$ with $P_{3} Q_{2}$ (yields $R_{3}$ ),

-intersect $P_{1} Q_{3}$ with $P_{3} Q_{1}$ (yields $R_{2}$ ),

-place a point in the direction $R_{2}$ on the line $R_{1} R_{3}$ (yields $R_{2}^{\prime}$ ).

At the conclusion of the experiment, we ended up with a pair of points $\left\{R_{2}, R_{2}^{\prime}\right\}$ in visual space (and thus in physical space!) that should coincide if Pappus is satisfied. Thus the verification of Pappus in visual space was reduced to the verification of the coincidence of two points in physical space, which is up to statistical test.

The configurations used in this experiment are depicted in Figure 1. Notice that they fill a very large part of the visual field in front of the observer and involve a rather large range. We decided to use such unusually extended configurations (at least when compared with the literature; in daily life the relevant visual field is usually quite large) because violations of the Pappus condition are much more likely to show up this way than when small configurations (more in

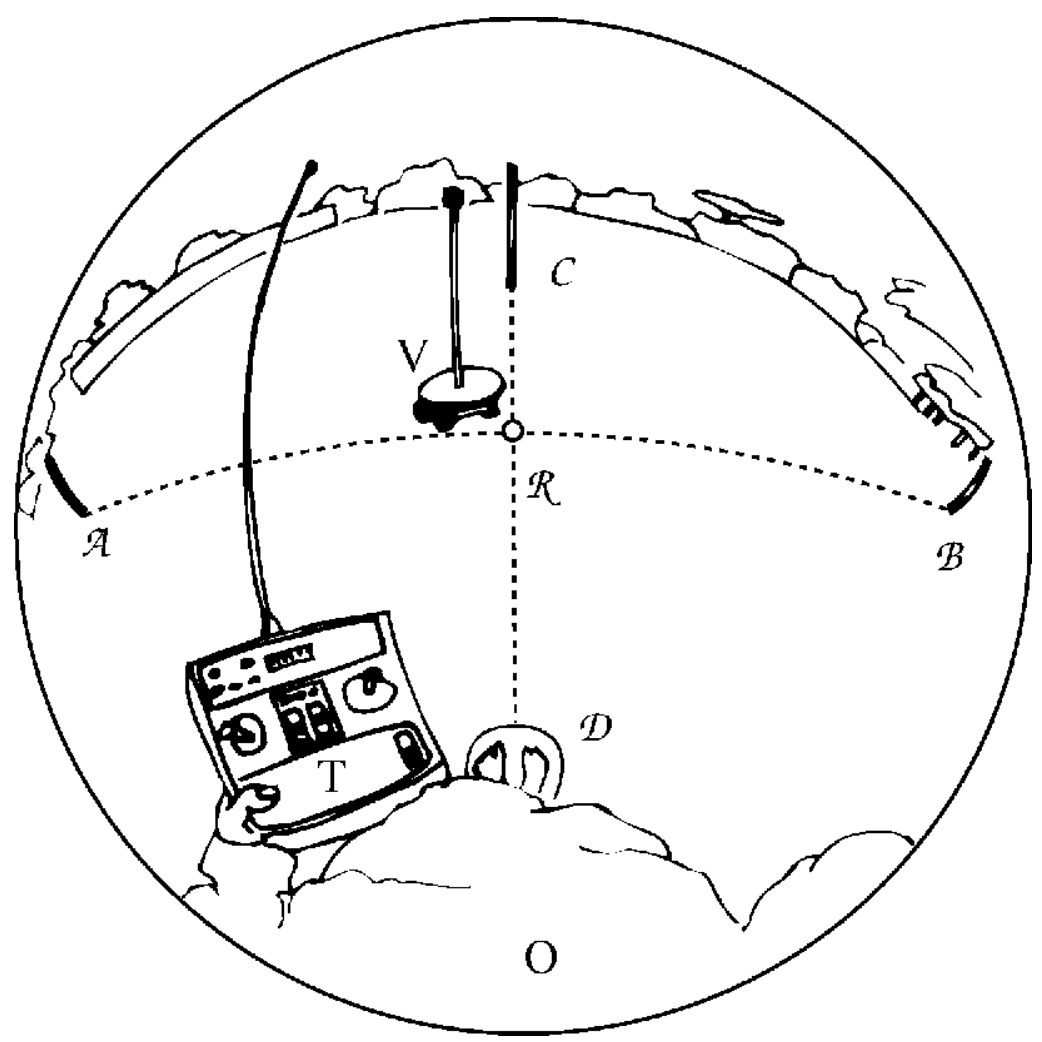

Figure 3. Tracing of a fish-eye photograph (a full $180^{\circ}$ field of view) taken from the observer's vantage point. The space was a lawn with distant trees and some buildings. In this figure, the task of the observer (at $O$, feet at $D$ ) was to move the cube on a pole on the vehicle $V$ to the intersection of the line defined by the stakes $A$ and $B$ with the egocentric direction toward stake $C$, say the point $R$. The observer guided the vehicle by remote control by means of the transmitter, $T$. 
line with conventional work) would be used. Two different configurations were tested; they differed only through the location of the observer. Sessions for the two configurations were alternated.

These configurations contained obvious regularities, but because the observer never saw the full configuration, these were not apparent in practice, as they are in Figure 1.

One session took about $1 \mathrm{~h}$. This included geodesy and the switching of stakes; the observer may have been actively involved for about half an hour. Each observer completed five sessions for each configuration. Thus, each observer spent a total of about $10 \mathrm{~h}$ at the task.

\section{RESULTS}

Three visually collinear points are rarely collinear in physical space. Since three points in physical space define a unique circle, we may conveniently indicate the empirically determined visual lines through circular arcs in physical space. (See Figure 4.) Thus we may visualize the configuration produced during a session in physical space. We found that although the results were typically similar from session to session (for a single observer), differences occurred, sometimes even in the sign of the curvatures when these were small. Also, the observers differed quantitatively among each other, though the results were qualitatively similar. The simplest analysis should at least address the issue of the systematic deviations as well as the violations of the Pappus configuration. The systematic deviations were with respect to the veridical setting (the Euclidean plane), whereas the violations of Pappus were the differences between $R_{2}$ and $R_{2}^{\prime}$. Thus, it is quite possible to have large systematic deviations with Pappus still being satisfied.

\section{Investigation of the Pappus Property}

In Figure 4, we have plotted the covariance ellipses for the various vertices of the two Pappus configurations in optical space on the basis of the data from all five sessions. The points $P_{1}, P_{3}, Q_{1}, Q_{3}$ and the observer positions are of course exactly known. All the other points $\left(P_{2}, Q_{2}\right.$ and $R_{1}$, $R_{2}$ and $R_{3}$ ) were reproduced various times by the observer. The arcs have been calculated for the mean positions. Of course, the scatter in the locations of these points is dependent, since a location is constructed from previous ones, so deviations are compounded. Thus the covariance ellipses do not give a fair perspective on the scatter. For instance, in the final test, one should compare $\left\|R_{2}-R_{2}^{\prime}\right\|$ (per session) with zero, rather than the means of $R_{2}$ and $R_{2}^{\prime}$ over the sessions with each other. One immediate informal observation is that — even in a single session - the points $R_{2}$ and $R_{2}^{\prime}$ tended to deviate rather markedly from the physical location, whereas they were generally closely bunched together. Thus the Pappus violation (remember that there always will be a violation, though not necessarily significant) is typically much smaller than the deviation from veridicality.

In Figure 5, we show all deviations of veridicality with $5 \%$ covariance ellipses. The filled point is the physical location, the open dots the observations $R_{2}$. Veridicality was generally violated at the $5 \%$ level, except for Observer
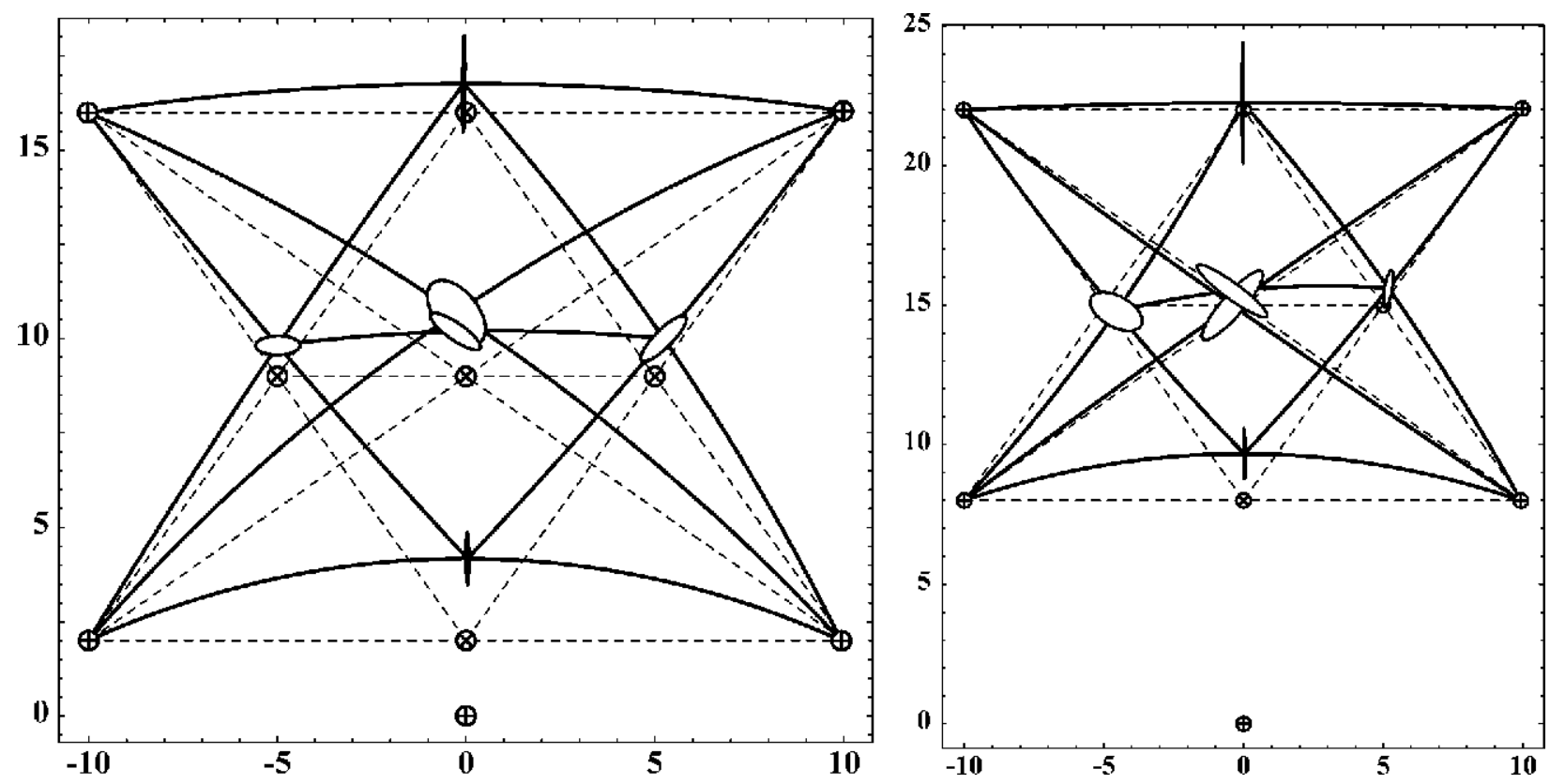

Figure 4. Results for observer S.P. in the near (left) and the far (right) conditions. The veridical configuration is indicated via crossed circles and dotted lines. The visual configuration is indicated via covariance ellipses and drawn lines. Notice that the visual configuration deviates from the veridical one, especially in the near condition. Pappus is not significantly violated when the covariance ellipses for the points $R_{2}$ and $\boldsymbol{R}_{2}^{\prime}$ overlap. Notice that this is the case, although deviations from veridicality in the near condition are quite significant. All measures are in meters. 
E.T.
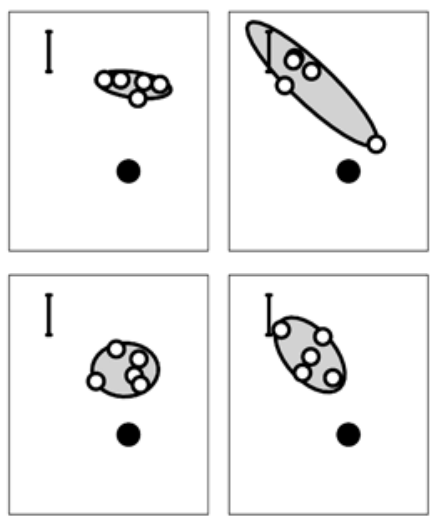

R.H.
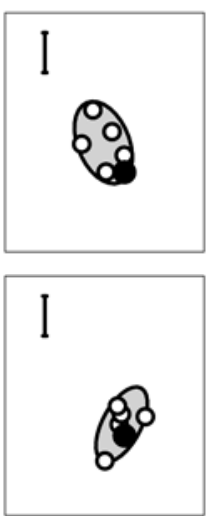

S.P.

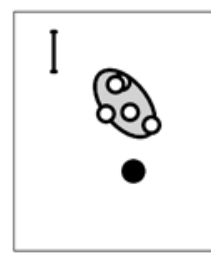

near

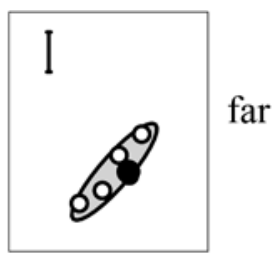

Figure 5. All deviations from veridicality with $5 \%$ covariance ellipses. The filled points are the physical locations, the open dots the observations, $R_{2}$. Veridicality was violated at the $5 \%$ level, except for Observer R.H., who was nearly veridical throughout, and Observer S.P. in the far condition. Notice the scale. The indicated stretch is $1 \mathrm{~m}$. Thus the point $R_{2}$ can easily be several meters "off."

R.H., who was nearly veridical throughout, and Observer S.P. in the far condition. Notice the scale: The indicated stretch is $1 \mathrm{~m}$. Thus the point $R_{2}$ can easily be several meters "off." Optical space is quite different from physical space, even in our full-cue situation.

A direct test of Pappus violations is to check whether the observations of the differences $R_{2}-R_{2}^{\prime}$ are statistically nonvanishing. In Figure 6, we show all data with $5 \%$ covariance ellipses. In only one case (Observer H.N. in the far condition) did we find a statistically (just) significant violation; in all other cases, Pappus was not violated. Thus, optical space was projective within the resolving power of our method.

In Figure 7, we show an overview of all relevant data. The deviations from veridical were significant in all except one case (Observer S.P. in the far condition). In the near condition, these deviations were strikingly large, and in the far condition-although smaller for all observers than in the near condition - they tended to be appreciable. In all cases, the violation of the Pappus condition was much smaller (in the absolute sense) than the deviation from veridicality, except in the noted case in which the deviation from veridicality itself was not significant. As shown earlier, these violations were not significant on the 5\% level.

It is not possible to say whether the number of violations would increase if the number of repeats were increased. In the case where the violations would "really" be zero, the size of the covariance ellipses would decrease, but the ellipse would still contain the origin. In the case where there were "really" a (necessarily small) violation, the decrease of the size of the covariance ellipse would eventually reveal the violation. Given the finite data volume, we cannot decide.

Thus, one robust conclusion is certainly that optical space deviates markedly from physical space, but that in spite of that, the Pappus condition holds up surprisingly well. Notice that this is a stronger conclusion than nonviolation of Pappus for overall veridical results (as might perhaps have been expected given the full-cue situation).

A summary of the data is presented in Table 1. A formal test of significance of the difference of the means of the magnitudes (absolute values) of the deviation from veridicality and the violations of the Pappus condition (again, absolute values) reveals that in all cases where the deviation was significant (all but one case), the Pappus violation was significantly smaller than this deviation. The ratios were quite large, about an order of magnitude in the near condition, systematically less in the far condition. Notice that all observers produced qualitatively equivalent results, though quantitative differences were certainly significant.

We clearly have to reject the hypothesis that human visual space has an empirically detectable noncommutative structure (Pappus consistently violated), whereas we have to accept violations of veridicality.

\section{Apparent Frontoparallels}

As a by-product of this work we have collected four empirical frontoparallels (i.e., $P_{1}-P_{2}-P_{3}$ and $Q_{1}-Q_{2}-Q_{3}$ in both the near and far conditions) for our 4 observers (see Figure 8). This is of some interest by itself because such entities have rarely been reported before in open-field conditions (Battro, di Piero Netto, \& Rozestraten, 1976). The empirical frontoparallels for pure binocular stereopsis (socalled Helmholtz horopters) have only been obtained in near space and for limited angles of view (Ames, Ogle, Glidden, 1932; Helmholtz, 1866; Ogle, 1950). They look quite different from our results. Note how remarkably close to veridical these frontoparallels really are, perhaps surprising in view of the very large fields of view (up to $160^{\circ}$ ) in- 
E.T.
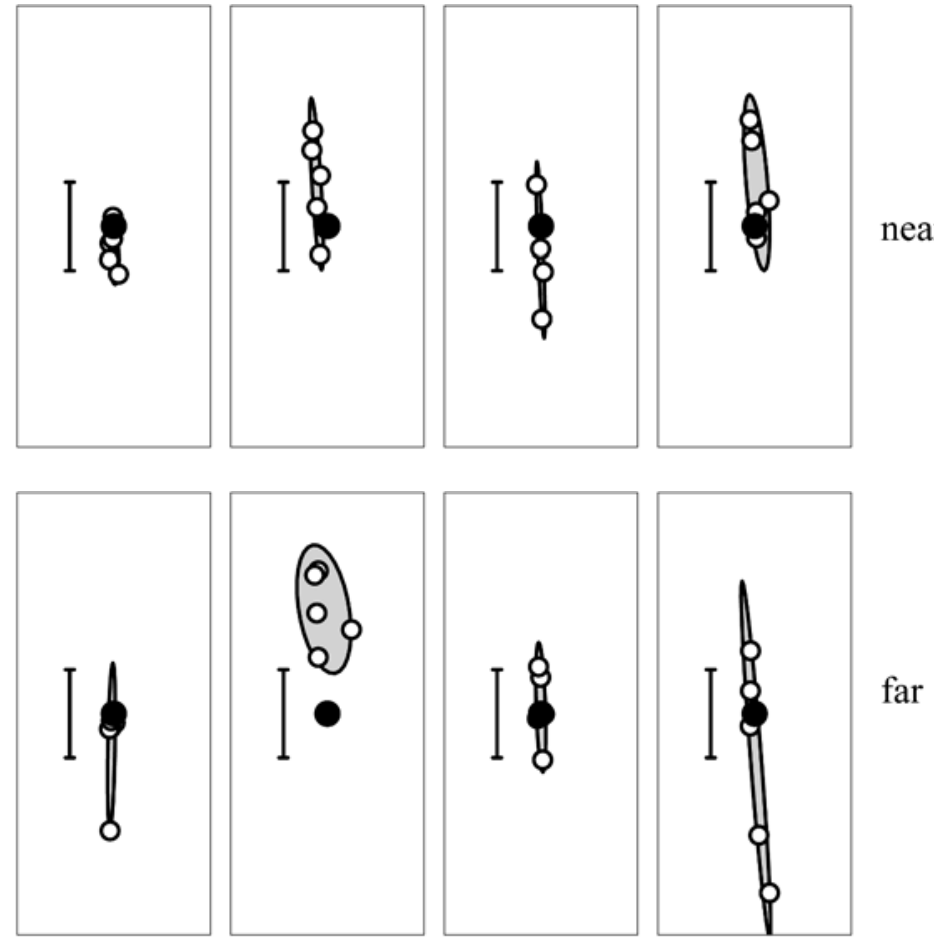

Figure 6. All values of the violation $R_{2}-R_{2}^{\prime}$ collected in the experiment. Open circles are datapoints, the closed circle denotes the origin (null hypothesis). The ellipses denote the $5 \%$ probability contours. Only in one case (Observer H.N. in the far condition) was a violation detected. Notice the scale. The indicated stretch is $1 \mathrm{~m}$. Average violations were typically small, certainly less than $1 \mathrm{~m}$.

Near

Far

E.T. |ํำ<smiles>O[Tl]1CO1</smiles><smiles>C[AlH]O</smiles>

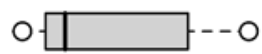

H.N. $\mid q \square$ -
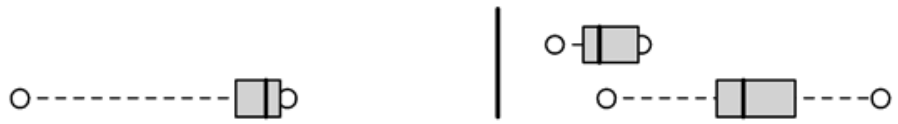

R.H.
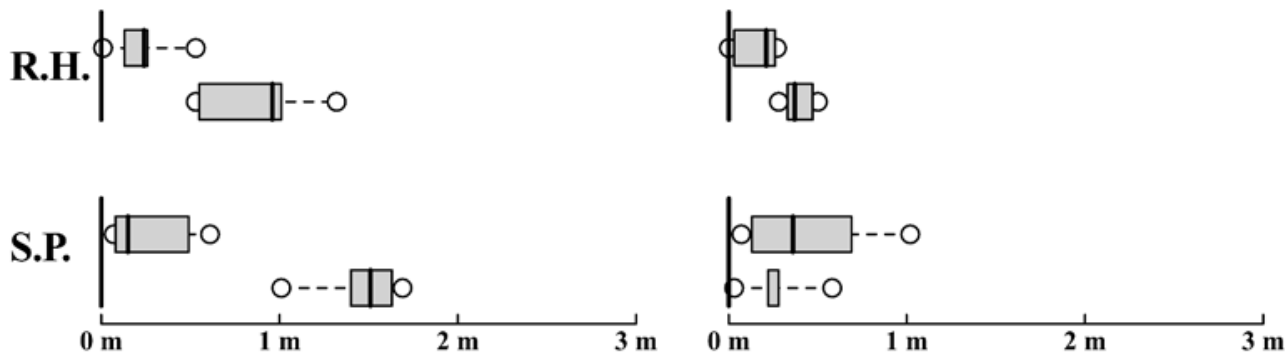

Figure 7. An overview of all results. The absolute values of the deviations from veridicality (lower row in each subfigure), and the violation of Pappus (upper row in each subfigure) are indicated via their median, interquartile range, and total variation. Notice that the deviations from veridicality were generally large, the violations much smaller. 
Table 1

Mean Absolute Deviation From Veridicality and Mean Absolute Violation of the Pappus Condition (in Centimeters) for all Observer-Distance Combinations

\begin{tabular}{cccccc}
\hline & & & & & Pappus \\
Observer & Distance & Deviation & Violation & Ratio & Violation \\
\hline E.T. & near & 217 & 14 & $16.0(+)$ & no \\
H.N. & near & 219 & 31 & $7.2(+)$ & no \\
R.H. & near & 74 & 23 & $3.2(+)$ & no \\
S.P. & near & 170 & 28 & $6.1(+)$ & no \\
E.T. & far & 162 & 18 & $8.8(+)$ & no \\
H.N. & far & 200 & 59 & $3.4(+)$ & yes \\
R.H. & far & 53 & 15 & $3.5(+)$ & no \\
S.P. & far & 54 & 45 & $1.2(-)$ & no \\
\hline
\end{tabular}

Note-Also given are the ratio of the deviation from veridicality to the Pappus violation (both absolute) and the significance of the difference between the deviation and the violation (both absolute; "+," significant at the 5\% level; "-," not significant at the 5\% level). Notice that the ratios are generally high, especially in the near condition, thus the violations are typically much less than the deviations from veridicality. In the one "draw" case (observer S.P. in the far condition), the response was essentially veridical. In the final column, we show the results of a test of violation of the Pappus condition. The null hypothesis is that $\left\|R_{2}-R_{2}^{\prime}\right\|=0$; we test at the 5\% level. In only one case (observer H.N. in the far condition) was the Pappus condition significantly violated.

volved. Even when head and body movements were a prerequisite for doing the task, the observers were quite close to veridical.

The results in the literature that perhaps come closest are Battro et al.'s (1976). These authors reported Helmholtz horopters for $12^{\circ}$ width, at distances ranging from .5 to $45 \mathrm{~m}$. Some of their subjects produced results that are quite similar to ours, but others showed several sign transitions (concave/convex transitions), thus greatly encumbering the interpretation of their results.

\section{CONCLUSIONS}

In this paper, we investigated the projective structure of visual space. This involved a "step back" from the position taken by the authors of the classical literature who have taken the projective structure essentially for granted, with few exceptions. For instance, the following quote by Blank (1958a) is typical:

On the basis of single qualitative tests on a number of observers it is postulated that the binocular visual space is adequately described as a Riemannian space of constant Gaussian curvature. In order to categorize the intrinsic visual geometry it is then necessary to determine the sign of the curvature, ... This problem is left to experiment.

Here Blank even takes the Riemannian metrical structure for granted and leaves only the determination of the curvature to psychophysics. Most authors indeed rely on metrical judgments without much ado (e.g., "The perceptions he is asked to report . . . are of three rudimentary kinds, equality of distance, straightness, and perpendicularity; there are no others" [Blank, 1958b], or "This paper presents a systematic investigation of judgments of distances, angles and areas - the metric properties of space" [Wagner, 1985]. In this work, we totally abstain from metrical judgments (distances, angles, areas, perpendicularity); the only (really rudimentary) judgments we allow are incidences of points and lines.

We found strong and systematic deviations from veridicality but no obvious systematic violations of the Pappus property. We consider the absence of obvious violations in such a highly deformed empirical configuration to be a strong result. Of course, we were in no position (given realistic temporal limits) to explore the Pappus property for more than a few configurations (see note 2 ). It would be definitely interesting to include both very different configurations (if when one generates random Pappus configurations there turns out to exist a number of qualitatively quite different configurations) and different locations of the observer with respect to these configurations.

It might be argued that the structure of the ground plane is simply the structure of the visual field and that the task could be performed entirely in the visual field. Then, the observer could ignore the horizontal plane at eye height

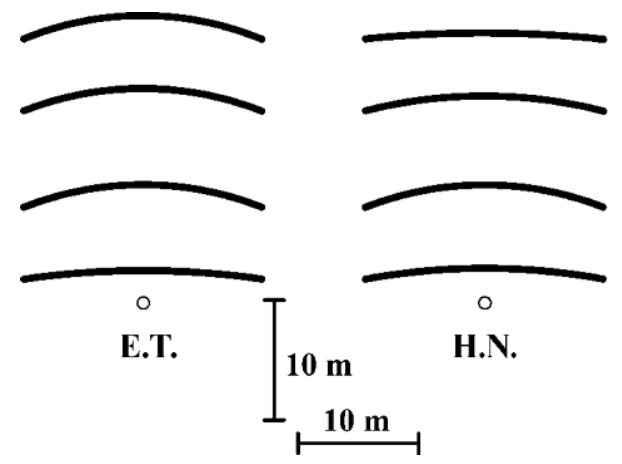

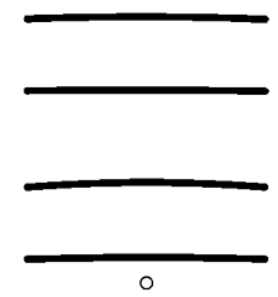

R.H.

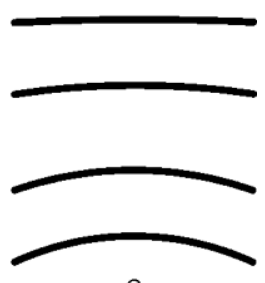

○

S.P.

Figure 8. The apparent frontoparallels for all observers. Notice that the visual field was in all cases much larger than those that apply to results in the literature (e.g., the Helmholtz horopters), thus a meaningful comparison is not really possible. 
altogether and could merely attend to the footpoints of the markers and the vehicle. Distances would play no role then. Although this is indeed a priori a reasonable idea, our results clearly indicate otherwise. The observers judged relations in their visual world - that is to say, both in the visual field and in the depth domain. Upon debriefing, all observers were convinced that the aim of the experiment had to do with the veridicality of their distance judgments, even though our explicit instructions mentioned only intersection. We instructed the observers to attend to a mark on a pole mounted on the vehicle at about $1.55 \mathrm{~m}$ above ground level, rather than the ground location of the vehicle. But the "depth" is evidently derived in large part from these ground locations. It is the total scene in front of the observer that determines the layout of the visual world (as opposed to the visual field); herein lies the difference with the classical work with luminous points in dark rooms.

The known structure of the visual field (subjective curvatures of straight lines; Hauck, 1879; Helmholtz, 1866; Pirenne, 1970) does not explain our findings at all. Most observers perceive objective straight lines in the visual field as curved, if these lines are not concurrent with the center of the visual field. Thus, such subjective curvatures should vanish when a point on that line is being fixated (Helmholtz, 1866). Our observers invariably looked at the cube on top of the pole on the vehicle when checking the result of their efforts; thus, any influence of the subjective curvatures is out of the question.

The empirical frontoparallels reported here deviated markedly from frontoparallels reported by us (Koenderink, van Doorn, \& Lappin, 2000) that were obtained with an exocentric pointing task. These results were obtained under rather similar conditions (open field, large fields of view), although the task was quite different. The conclusion has to be that the results depend quite strongly on the precise operationalization. This appears to open an important field of future research.

In conclusion, then, we have found that in the projective structure of the horizontal plane at eye height, the Pappus configuration is consistent, not withstanding the fact that the geodesics of visual space are physically significantly curved. Perhaps surprisingly, these curvatures are quite unlike the classical curvatures in visual space or in the visual field. Indeed, their sense is opposite to that of the classical (Ogle, 1950) Helmholtz horopters. This is a highly significant and unexpected finding. It is doubtless due to the fact that we worked under full-cue conditions, rather than with punctate targets in an empty field. Although we interpret our results in terms of the structure of the horizontal plane at eye height, it must be kept in mind that this plane is embedded in the visual world of the observer and derives its very structure from that. Signs of this were apparent from certain left-right asymmetries in the empirical Pappus configuration (see Figure 4, right panel; this occurred for all observers), which we believe to have been due to the asymmetry in the scene (group of dark trees to the right, a building to the left of the observer).
Since the horizontal plane at eye height, though not Euclidean, has a tight projective structure, this opens the way to check its possible affine properties.

\section{REFERENCES}

Ames, A. Ogle, K. N., \& Glidden, G. H. (1932). Corresponding retinal points, the horopter and size and shape of ocular images. Journal of the Optical Society of America, 22, 538-631.

Battro, A. M., Di Piero Netto, S., \& Rozestraten, R. J. A. (1976). Riemannian geometries of variable curvature in visual space: Visual alleys, horopters, and triangles in big open fields. Perception, 5, 9-23.

Beltrami, E. (1865). Risoluzione del problema: Riportare i punti di una superficie sopra un piano in modo che le linee geodetiche vengano rappesentate da linee rette [Solution to the problem: Find the embedding of a surface such that the parameter curves are geodesics]. Annali di Matematica Pura ed Applicata, ser. 1, 7, 185-204.

Beltrami, E. (1868). Saggio di interpretazione della geometria noneuclidea [On the meaning of non-Euclidean geometry]. Giornale di Matematiche, ad uso degli studenti delle università italiane, $\mathbf{6}$, 284-312.

BENNETt, M. K. (1995). Affine and projective geometry. New York: Wiley. Blank, A. A. (1953). The Luneburg theory of binocular visual space. Journal of the Optical Society of America, 43, 717-727.

BLANK. A. A. (1958a). Analysis of experiments in binocular space perception. Journal of the Optical Society of America, 48, 911-925.

BLANK, A. A. (1958b). Axiomatics of binocular vision. The foundations of metric geometry in relation to space perception. Journal of the $\mathrm{Op}$ tical Society of America, 48, 328-334.

Blumenfeld, W. (1913). Untersuchungen über die scheinbare Grösse im Sehräume [Investigations on apparent size in visual spaces]. Zeitschrift für Psychologie, 65, 241-404.

Blumenthal, L. M. (1995). A modern view of geometry. New York: Dover. CoXeter, H. S. M. (1961). Introduction to geometry. New York: Wiley.

FoLEY, J. M. (1964). Desarguesian property of visual space. Journal of the Optical Society of America, 54, 684-692.

GiBson, J. J. (1950). The perception of the visual world. Boston: Houghton Mifflin.

HaUck, G. (1879). Die subjektive Perspektive und die horizontalen Curvaturen des Dorischen Styls [Subjective perspective and the curvature of horizontals in the Doric style]. Stuttgart, Germany: K. Wittwer.

Helmholtz, H. von (1866). Handbuch der physiologischen Optik [Handbook of physiological optics] (1st ed.). Hamburg and Leipzig: Leopold Voss.

Hilbert, D., \& Cohn-Vossen, S. (1983). Geometry and the imagination. New York: Chelsea. (Original work published 1932)

Hillebrand, F. (1929). Lehre von den Gesichtsempfindungen aufGrund hinterlassener Aufzeichnungen (Herausgegeben von Dr. F. Hillebrand) [Studies in visual perception from the heritage of Hillebrand, communicated by F. Hillebrand]. Wien: Springer-Verlag.

InDow, T. (1990). On geometrical analysis of global structure of visual space. In H.-G. Geissler \& M. H. Miller (Eds.), Psychophysical explorations of mental structures (pp. 172-180). Göttingen: Hogrefe \& Huber.

InDOw, T. (1991). A critical review of Luneburg's model with regard to global structure of visual space. Psychological Review, 98, 430-453.

INDOw, T. (1997). Hyperbolic representation of global structure of visual space. Journal of Mathematical Psychology, 14, 89-98.

KLEIN, E. (1932). Elementary mathematics from an advanced standpoint: Arithmetic, algebra, analysis (E. R. Hedrick \& C. A. Noble, Trans.). New York: Macmillan.

KLEIN, E. (1939). Elementary mathematics from an advanced standpoint: Geometry (E. R. Hedrick \& C. A. Noble, Trans.). New York: Macmillan.

Koenderink, J. J., van Doorn, A. J., \& LAPpin, J. S. (2000). Direct measurement of the curvature of visual space. Perception, 29, 69-80.

Luneburg, R. K. (1947). Mathematical analysis of binocular vision. Princeton, NJ: Princeton University Press.

Ogle, K. N. (1950). Researches in binocular vision. Philadelphia and London: W. B. Saunders. 
Pirenne, M. H. (1970). Optics, painting and photography. Cambridge: Cambridge University Press.

Suppes, P. (1977). Is visual space Euclidean? Synthese, 35, 397-421.

Wagner, M. (1985). The metric of visual space. Perception \& Psychophysics, 38, 483-495.

\section{NOTES}

1. Both the Desargues and Pappus properties relate to "rare" cases of incidence, such as three distinct points being collinear (incident with a single line) or three distinct lines being concurrent (incident with a single point). If one considers configurations of "random" elements, such incidences have a probability of zero. If one has a configuration that is very "special," in the sense that it contains a number of such rare incidences, the Desargues and Pappus properties allow one to construct yet another one. For instance, the Desargues property states that if two triangles are point perspective (there exists a point that is collinear with any pair of vertices), they are also line perspective (there exists a line that is concurrent with any pair of sides) and vice versa. The Pappus property allows one to construct a collinear triple of points, given any two collinear triples.

2. We obviously cannot generalize from this work and state that the Pappus property is generally valid. That would mean checking an infinite number of configurations.

(Manuscript received September 20, 2000; revision accepted for publication July 2, 2001.) 